\section{Ueber die therapeutischen Erfolge mit der direkten Magenelektrisation.}

\section{Von Dr. Max Einhorn,}

Arzt am Deutschen Dispensary und Privatdocenten an der N.-Y. Postgraduate Medical School, New-York.

(Fortsetzung aus No. 33.)

Nach dieser längeren Abschweifung möchte ich zum eigentlichen Thema zurückkehren, nämlich zu der therapeutischen Wirksamkeit der direkten Magenelektrisation.

\section{A. Die direkte Magenfaradisation.}

Wie in meiner früheren Arbeit angegeben, wurde von einzelnen Autoren die direkte Elektrisation des Magens ab und zu vorgenommen, allein ziemlich selten, und liegen in der Litteratur fast gar keine detaillirten Angaben über die direkte Magenfaradisation vor. - Ich betrachte es als ein grosses Verdienst von Charles G. Stockton, ${ }^{1}$ ) dass er consequent diese Behandlungsmethode durchführte und in dem American Journal of the Medical Sciences veröffentlichte. Stockton hat die direkte Faradisation des Magens in mehreren Fällen von Magendilatation, ferner in einigen Fällen von functionellen Störungen des Magens, mit und ohne gesteigerte Acidität, mit grossem Erfolge angewandt. In dieser Arbeit sucht Stockton die Wirkung der Elektrisation zu erklären und sagt dabei folgendes: "Genau die Rolle anzugeben, welche die Faradisation spielt, vermag ich nicht; ich bin ungewiss ob dieselbe als ein sedatives oder ein stimulirendes Magenmitte aufgefasst werden kann .... Die Erfolge waren so günstig, dass ich den faradischen Strom in Fällen, die von ganz entgegengesetztem Charakter waren, angwandt habe, und ich schloss gerade aus dem gemeinschaftlichen Erfolge, dass die grosse Mannichfaltigkeit der gastrischen Neurosen auf einer gemeinsamen Ursache basirt - auf einer unvollkommenen Innervation des Magens; die Elektricität verbessert nun die Innervation und entfernt so die Ursache und mit ihr jene Krankheitszustände, welche auf den ersten Blick sich so entgegengesetzt gegenüberstehen."

Da der Gegenstand ziemlich neu und für die Praxis wichtig ist, werde ich mir erlauben, eine hinlängliche Anzahl von mit der direkten Magenfaradisation behandelte Fällen als Repräsentanten der verschiedenen Krankheitsformen anzuführen und genauer zu besprechen. Die Applicationsmethode war die, welche ich in der Berliner klin. Wochenschrift 1891, No. 23 beschrieben habe; der Strom wurde nur so stark gewahlt, dass deutliche Contractionen der Bauchdecken ausgelöst wurden, ohne dass erhebliche Schmerzen vom Patienten empfunden wurden; die Dauer der Sitzung war stets zehn Minuten.

Von Juni 1890 bis November 1891 habe ich Gelegenheit gehabt, die direkte Magenfaradisation bei 29 Patienten curmässig anzuwenden. Diese vertheilten sichauf die einzelnen Magenerkrankungen wie folgt:

Hyperacidität mit Dilatation

(davon drei mit heftigen Gastralgieen und einer mit continuirlicher Hypersecretion)

Gastroxynsis .

Aufstossen, Atonie der Cardia (?)

Gastritis glandularis chronica

Gastritis glandularis chronica mit Fehlen der freien Salzsäure-

beginnende Atrophie der Magenschleimhaut)

Anadenia ventriculi (Atrophie der Magenschleimhaut)

Hartnäckige Gastralgieen

(darunter einer mit Gastrosuccorrhoea chronica continua).

\section{Fälle von Hyperacidität.}

Fall 1. 12. März 1891. H. J. F., etwa 33 Jahre alter Kaufmann, leidet seit drei Jahren an Schmerzen nach dem Essen; seit einigen Monaten haben die Beschwerden nach dem Essen derart zugenommen, dass Patient genöthigt war, nach jedem Essen Erbrechen hervorzurufen, um sich dadurch zu erleichtern. Der Appetit blieb ganz weg.

1) Stockton, American Journal of the Medical Sciences 1890, p. 20.
Bei der Untersuchung wird die untere Magengrenze in der Mitte zwischen Nabel und Symphyse festgestellt.

13. Mărz 1891. Die Untersuchung des Mageninhalts eine Stunde nach dem Probefruhstück ergiebt: $\mathrm{HCl}+$ Acid $=96$.

Diagnose: Dilatatio ventriculi, Hyperaciditas, Gastralgia.

Neben der gewöhnlichen Behandlung mit Alkalien wird Patient direkt gastrofaradisirt. - Patient giebt bereits nach der dritten elektrischen Sitzung an, dass die Schmerzen weniger geworden seien, und dass er an den Tagen, wo er faradisirt wird, mit gutem Appetit essen kann. Im Verlauf von einem Monat hat sich der Zustand des Patienten soweit gebessert, dass er keine Schmerzen hatte und gut essen konnte.

Laut einem Briefe, den ich vom Patienten am 24. October 1891 erhielt, bat derselbe sich seitdem frei von Schmerzen gefthlt

Fall 2. 15. August 1890. J. K., 36 Jahre alt, leidet seit drei Jahren an hartnäckiger Verstopfung, dabei sind Kopfschmerzen vorhanden. Gefühl von Vollsein im Magen. Kein Erbrechen. Aufstossen auch von Wasser immer vorhanden, so dass sein Hals selbst dadurch rauh wurde. Hat 45 Pfund verloren.

Untersuchung: Die untere Magengrenze liegt zwei bis drei Finger unterhalb des Nabels.

16. September 1890. Nüchtern: Magensaft $60 \mathrm{ccm}: \mathrm{HCl}+$, Acid. -990 . Biuretreaction + , Spur.

18. September 1890. Eine Stunde nach Probefruhstuck: $\mathrm{HCl}+$, Acid. $=100$.

Diagnose: Gastrosuccorrhoea continua chronica; Hyperacidität; Dilatation.

Therapie: Magnes. ust., Pulv. rad. Rhei ana 7,5, Natr. bicarb., Sacch. lact. ana 25,0, dreimal täglich einen Theelöffel voll und Magenausspülungen.

Nach der alkalischen Behandlung bessert sich der Zustand des $\mathrm{Pa}$ tienten, doch bleibt noch immer ein Geftuhl von Vollsein zurtick. Der Magen wird direkt faradisirt; nach einigen Sitzungen giebt Patient an, sich erheblich leichter zu fuhlen; Patient nimmt zu, und die Beschwerden nach den Mahlzeiten bleiben weg. Die Hyperacidität sowie die Hypersecretion blieben jedoch, wenn auch in etwas geringerem Maass weiter bestehen.

Fall 3. 30 Jahre alter Kaufmann, leidet seit mehreren Jahren an Verdanungsbeschwerden, A ppetit nicht vorhanden; Aufstossen recht viel; Schmerzen ab und zu; Gefühl der Völle nach den Mahlzeiten; Verstopfung.

Untersuchung ergiebt: untere Magengrenze drei bis vier fingerbreit unter dem Nabel (Gastrodiaphanie); daselbst kann man leicht Klatschgeräusche hervorrufen.

Eine Stunde nach dem Probefruhstuck: $\mathrm{HCl}+$, Acid $=120$. Behandlung: Alkalien und direkte Faradisation des Magens (den 26. April 1891)

Gleich nach den ersten Tagen der Behandlung bessert sich der Zustand des Patientin; er kann gut essen, Stuhlgang regelmässig; Aufstossen verringert. Am 26. April giebt Patient an, frei von Beschwerden zu sein.

Acht andere Fälle von Magendilatation mit Hyperacidität verbunden, ähneln so sehr den eben beschriebenen Fällen, sowohl was die krankhaften Symptome als auch was die Behandlung und den Einfluss der Faradisation anbetrifft, dass ich von einer genaueren Beschreibung derselben absehen kann.

$$
\text { II. Gastroxynsis. }
$$

31. August 1891. G. N. J., 42 Jahre alt, Kaufmann, leidet seit fünf Jahren an hăufig auftretenden Schmerzattaquen in der Magengegend, die mit Erbrechen von hochsauren Massen gewöhnlich einhergehen; die Anfälle kommen alle 3-4 Wochen einmal vor und halten etwa drei Tage an; während des Anfalls ist Patient elend und niedergeschlagen, hat heftige Schmerzen, kann kaum etwas geniessen und muss ofter brechen. Nach uberstandenem Anfall ftihlt sich Patient ganz gesund, nur ist der Schlaf nicht sehr gut.

Die Untersuchung ergab: Brust- und Abdominalorgane normal. Kniereflexe vorhanden. Magen nicht erweitert, durch die Gastrodiaphanie festgestellt.

31. August 1891. Eine Stunde nach dem Probefrühstück: $\mathrm{HCl}+$ Acid. $=66$.

Diagnose: Gastroxynsis. Therapie: Patient wurde während des Monats September einen Tag um den anderen und während des October zweimal wöchentlich gastrofaradisirt.

Patient hat weder während der Behandlungszeit noch nachher irgend einen Anfall seitens seines Magens gehabt, schläft gut und fuhlt sich jetzt kräftiger und lebensfroher.

\section{Fälle von Aufstossen.}

Aufstossen ist ein Symptom, das bei allen möglichen Störungen der Magenfunction gelegentlich auftritt. Hier sollen jedoch einige Fälle besprochen werden, wo dieses Symptom so in den Vordergrund trat, dass die anderen Beschwerden des Patienten dadurch in den Schatten gestellt wurden.

Fall 1. 7 October 1890. Henry I etwa 40 Jahre alt, leidet seit einigen Jahren an häufigem Aufstossen: im letzten Jahre nahm das Aufstossen so überhand, dass er dadurch in seinem Geschäfte vielfach behindert ist; ausserdem verspürt Patient seit einigen Monaten ein $\mathrm{Ge}$ fühl von Vollsein nach .dem Essen und Mudigkeit; Appetit mässig, Stuhlgang etwas angehalten.

Status praesens: Brustorgane intact; bei der Palpation des Abdomens fallen Klatschgeräusche in der Magengegend auf; dieselben können bis zweifingerbreit oberhalb der Nabellinie erzeugt werden. 
10. October 1890. Eine Stunde nach dem Probefrühstuck: $\mathrm{HCl}+$ Acid $=80$.

Diagnose: Hyperaciditas und Atonie (?) der Cardia. Behandlung: Magnes ust., Pulv. rad. Rhei. ana 5,0, Natr. carbon., Natr. bicarbon., Sacch. lact. ana 15,0 $3 \mathrm{mal}$ tägl. 1. T. Ausserdem direkte Magenfaradisation zwei- bis dreimal wöchentlich. - Patient fühlte sich bereits nach einigen elektrischen Sitzungen bedeutend besser; am 6. November wurde die Behandlung eingestellt. - Patient gab mir später an, dass er seitdem fast gar nicht mehr von Aufstossen geplagt wurde.

Am 4. Juni 1891 suchte mich Patient wieder auf, weil er nach einer acuten Indigestion wieder Beschwerden in der Magengegend verspürte; der Appetit war verringert, und auch das Aufstossen war theilweise wiedergekehrt.

Vom 4. bis 19. Juni wurde bei dem Patienten wieder regelmässig zweimal wöchentlich die direkte Magenfaradisation vorgenommen; sodann nur einmal wöchentlich bis zum 27. Juli 1891. Die Beschwerden des Patienten, namentlich das Aufstossen, verschwanden bereits in den ersten 14 Tagen der Behandlung; doch wurde die Cur der Vorsicht halber ein wenig weiter ausgedehnt. Seitdem ist Patient von seinen Beschwerden frei geblieben.

Fall 2. Frl. E. M., etwa 24 Jahre alt, leidet seit drei Jahren an Appetitlosigkeit, Aufstossen, Verstopfung und schlechtem Schlaf. Erbrechen war nicht vorhanden, dagegen Hochkommen von saurer Flüssigkeit nach dem Essen. Die Hauptbeschwerden der Patientin beziehen sich auf den sehr belästigenden Ructus, der ihr keine Ruhe lasst; sie wird durch das Aufstossen in ihrer Beschättigung gestört und muss vielfach infolge dessen jede Gesellschaft meiden.

1. April 1891. Status praesens: Zunge belegt, Klatschgeräusch am Magen, zwei fingerbreit unter dem Nabel; die rechte Niere deutlich palpabel und leicht verschiebbar. Die Untersuchung im ntichternen Zustande ergiebt: Gallenflüssigkeit im Magen, vermischt mit Magensaft; auch nach dem Probefrühstick wurde der Inhalt gewöhnlich mit Galle vermengt vorgefunden.

Die Untersuchung, eine Stunde nach dem Probefrühstlick, ergab am 3. April 1891: $\mathrm{HCl}+$, Acid. = 68; Gallenbeimengung.

5. April 1891. Im nüchternen Zustande finden sich im Magen etwa $70 \mathrm{ccm}$ von Galle gelblich gefärbt. $\mathrm{Fl}$ : $\mathrm{HCl}+$

Diagnose: Enteroptose, Acidităt nur wenig erhöht, Hypersecretion; Erschlaffung (Atonie) der Cardia und des Pylorus.

Therapie: direkte Magenfaradisation und gelegentliche Magenausspullungen.

Während des Monats April 1891 wurde Patientin einen Tag um den anderen faradisirt; die Ausspülung wurde einmal wöchentlich im nüchteren Zustande der Patientin vorgenommen; dabei wurde stets zuerst mit dem Schlauch festgestellt, ob der Magen irgend welchen Inhalt enthielt. Wăhrend des Monats April wurde dabei jedesmal die Anwesenheit einer grösseren Menge mit Galle vermischten Magensaftes im nüchternen Magen nachgewiesen. Nachher jedoch wurde die. Flissigkeitsmenge geringer, und im Juni wurde der Magen entweder leer vorgefunden, oder nur wenige Cubikcentimeter Magensaft, aber ohne Gallenbeimengung.

Patientin fuhlte sich nach den ersten Tagen der Behandlung besser das Aufstossen verlor sich gegen Ende April beinahe vollständig, und Patientin konnte ohne Beschwerden und mit Appetit essen. Im Juni und Juli wurde sie noch einmal wöchentlich faradisirt und sodann aus der Cur entlassen. Patientin hatte wăhrend der Behandlungszeit mehrere Pfund zugenommen und blieb seitdem ziemlich frei von Beschwerden.

Fall 3. Frau H. G., etwa 43 Jahre alt, leidet seit mehreren Jahren an einem Gefühl von Völle, schlechtem Appetit, häufigem Aufstossen. Während die anderen Verdauungsbeschwerden öfter für grössere Zeitintervalle wegbleiben, ist dies mit dem Aufstossen nicht der Fall, und belästigt sie dieses Symptom am meisten.

Status praesens: Patientin gut genăhrt, von gesunder Gesichtsfarbe; Zunge leicht belegt. Brustorgane intact. Bei der Palpation des Abdomens kann nichts abnormens entdeckt werden, ausser vielleicht eine gewisse Empfindlichkeit der Regio gastrica und epigastrica auf Druck. Eine Stunde nach dem Probefruhstuck: der Mageninhalt mit viel

Schleim vermischt: $\mathrm{HCl}+$, Milchsäure + , Gesammtacidität $=50$.

Diagnose: Gastritis glandularis chronica, Atonie der Cardia.

The rapi e: Tr. nuc. vomic., Aq. Amygd. amar. ana und direkte Faradisation des Magens, zuerst jeden anderen Tag, dann zweimal wöchentlich vom 16. September bis 23. October 1891. Nach einer Woche der Behandlung erhebliche Besserung, insofern als der Appetit gut wurde, und das Aufstossen sich verringerte. Am 23. October hatte Patientin keinerlei Beschwerden seitens des Verdauungsapparates, und das Aufstossen wa beinahe ganz verschwunden. Die Zunge sah rein aus, und die Untersuchung des Mageninhalts eine Stunde nach dem Probefrühstück ergab: $\mathrm{HCl}+$, Milchsäure $=0$, Acid. $=60$, die Schleimmenge äusserst gering

\section{Fälle von chronischem Magenkatarrh (Gastritis} glandularis chronica)

Ausser dem eben unter der Rubrik "Aufstossen" angeführten Fall von chronischem Magenkatarrh kamen noch vier Fälle dieser Erkrankung zur Behandlung mittels der direkten Magenfaradisation.

Einer dieser Fälle betraf Capitän L. M., der an Neurasthenie und chronischem Magenkatarrh, verbunden mit Dilatation litt; die direkte Magenfaradisation wurde hier einen Monat hindurch regelmässig angewandt, aber ohne irgend welchen Nutzen; sämmtliche Krankheitssymptome blieben unverändert bestehen. Die übrigen drei Fälle ähneln sich einander in jeder Hinsicht, und es genüge daher die Anführung eines Falles.
18. September 1891. Frau L. W., etwa 26 Jahre alt, litt seit ungefähr vier Jahren an häufig auftretenden Verdauungsbeschwerden (Appetitlosigkeit, Schmerzen in der Magengegend und im Leibe); sie wurde vielfach von verschiedenen Seiten betandelt, zuweilen mit Erfolg; den letzten Sommer über war ihr Wohlbefinden im allgemeinen gestört, doch haben ihre Beschwerden seit etwa sechs Wochen erheblich zugenommen; es stellten sich Schmerzen in der Magengegend ein; Patientin konnte nicht genügend essen, weil gleich nach Einnahme von Nahrung ein Gefühl sich einstellte, als ob sie zugeschnürt wäre; sie konnte nicht schlafen und hatte viel Aufstossen sowohl, wie Flatus, hatte an Körpergewicht bedeutend abgenommen.

Status praesens: Ziemlich blass aussehende, schmăchtige Person Farbe der Lippen und Wangen blass; Zunge belegt. Brustorgane intact. Die Bauchdecken schlaff, der Leib etwas aufgetrieben; die Regio epigastrica auf Druck etwas schmerzhaft; das Klatschgeräusch kann unter dem linken Rippenrande bis etwa drei fingerbreit oberhalb des Nabels erzeugt werden.

19. September 1891. Eine Stunde nach dem Probefrühstück: $\mathrm{HCl}=0$ Milchsäure +, Acid. $=60$, Schleim vorhanden; Erythrodextrin + , viel.

21. September. Nuchtern untersucht: Magen leer; bei der Auspulung wird nur wenig Schleim hinausbefördert.

Diagnose: Gastritis glandula chronica.

Therapie: Nux vomica, HCl, Gymnastik, kalte Abwaschungen und direkte Magenfaradisation; in den ersten 14 Tagen jeden anderen Tag, dann zweimal wochentlich. Die Faradisation wurde vom 23. September bis zum 20. October vorgenommen.

Patientin hatte sich gleich bedeutend besser gefühlt, sie konnte mehr essen, und die Schmerzen waren zuerst viel geringer, um bald beinahe ganz zu verschwinden. Patientin hat während der Behandlungszeit sechs bis sieben Pfund zugenommen und bekam eine gute, frische Gesichtsfarbe. Am 1. October ergab die Untersuchung des Mageninhalts eine Stunde nach dem Probefruhstuick: $\mathrm{HCl}+$, Milchsäure nicht vorhanden, Acid. $=56$ Die Salzsäure wurde nun weggelassen und statt dessen Arsenik und Eisen verabfolgt.

Am 23. October ergab die Untersuchung eine Stunde nach dem Probefruhstück: $\mathrm{HCl}+$, Milchsäure nicht vorhanden; Acid. $=60$.

Obgleich vom Magen aus jetzt keine Beschwerden da vorhanden waren, trat doch noch ab und zu (einmal in fün bis sieben Tagen) fưr kurze Zeit (etwa eine halbe Stunde) auf der linken Seite zweifingerbreit unter dem Rippenrande ein Schmerz auf, und es wurde deswegen bei der Patientin einmal wöchentlich der galvanische Strom angewandt, negativer Pol im Magen, die positive Elektrode auf die Schmerzstelle.

Am 2. November gab Patientin an, dass der Schmerz nach der Galvanisation nur einmal schwach aufgetreten sei.

V. Fälle von chronischem Magenkatarrh mit beginnender Atrophie der Schleimhaut.

Fall 1. J. K., 27 Jahre alt, leidet seit vier bis funf Jahren an schlechtem Appetit, Druck- und Vollegefuhl nach dem Essen; wird sehr viel von Aufstossen geplagt, Erbrechen nur ab und zu vorhanden; der Stuhl angehalten.

31. October 1890. Status praesens: Patient ziemlich mager; Brust intact; der Magen reicht bis zur Mitte zwischen Nabel und Symphyse (festgestellt durch die Gastrodiaphanie und durch Klatschgeräusche); auf Druck ist die Magengegend nicht besonders empfindlich.

2. November 1890 . Die Untersuchung des Mageninhaltes $1^{1 / 2}$ Stunde nach dem Probefruhstick ergiebt: $\mathrm{HCl}=0$, Milchsäure,+ Acid. $=30$.

12. November 1890. Eine Stunde nach dem Probefrühsttuck: $\mathrm{HCl}=0$, Acid. $=34$, Lab +. (Bei wiederholten Untersuchungen fand sich stets nach dem Probefrühstück dasselbe Resultat.)

Diagnose: Gastritis glandularis chronica, Atrophia incipiens; Dilatatio ventriculi.

Behandlung: Direkte Magenfaradisation (vom 31. October bis 16. November 1890 und vom 13. bis 26. April 1891) neben den anderen ublichen Vorschriften. Die Beschwerden des Patienten verloren sich schnell unter Behandlung, er bekam guten Appetit und hatte kein Aufstossen.

Wie bereits angefuhrt, war nach dem Probefrühstück im Magen keine freie Salzsäure nachweisbar. Es wurde am 16. November der Versuch gemacht, ob der Magen auf den faradischen Strom gentlgend $\mathrm{HCl}$ secernirt, um ihre Gegenwart nachweisen zu können. $\mathrm{Zu}$ diesem Zweck trank Patient im nüchternen Zustande $300 \mathrm{ccm}$ Wasser und wurde zehn Minuten gastrofaradisirt; nach 25 Minuten wurde ein Theil des Wassers aus dem Magen herausgeholt; durch Beimengung von Galle war dasselbe grünlich gefärbt; die Untersuchung ergab: $\mathrm{HCl}+$, Acid. $=16$.

Es gelang also auf diese Weise nachzuweisen, dass der faradische Strom bei dem Patienten, bei dem sich sonst keine freie Salzsäure vorfand, eine Magensaftproduction hervorrief, in der freie $\mathrm{HCl}$ vorhanden war.

Patient hatte sich während des ganzen Winters nach der Behandlung gut gefühlt, kam jedoch am 13. April, wo wieder Verdauungsbeschwerden aufgetreten waren, nochmals in Behandlung und konnte am 26. April 1891 als wieder gebessert entlassen werden. Das Verhalten des Magenchemismus hat sich gleichwohl nicht sichtlich geändert, insofern, als man auch jetzt bei dem Patienten eine Stunde nach dem Probefruh stück keine freie $\mathrm{HCl}$ vorfindet.

Fall 2. 14. März 1891. A. S., 32 Jahre, leidet seit drei Jahren an Verstopfung und Schmerzen in der Magengegend, ausstrahlend nach dem Rucken und den Schultern. Hat in den drei Jahren der Krankheit 15 Pfund verloren; hat niemals gebrochen, Appetit war immer gut.

Magen bedeutend erweitert, Klatschgeräusch vorhanden, herunterreichend bis zwei bis drei Finger unter dem Nabel. Schlaf gut, nacht spurt Patient nichts. Zunge etwas belegt. 
15. März 1891. Zwei Stunden nach dem Probefrühstück; beinahe die ganze Menge des eingenommenen Frühstïckes kommt heraus; $\mathrm{HCl}=0$, Acid. $=20, \mathrm{Lab}+$

Diagn os e: Gastritis glandularis chponica und Dilatation; beginnende Atrophie wahrscheinlich.

Neben der Verabreichung von $\mathrm{HCl}$ wurde Patient direkt gastrofaradisirt (vom 15. März bis 19. April 1891). Der Zustand besserte sich, insofern, als die Schmerzen verschwanden, allein die Untersuchung des Mageninhaltes ergab $1 \frac{1}{2}$ Stunden nach dem Probefrühstück am 17. April 1891: $\mathrm{HCl}=0$, Acid. $=22$, Lab + , also keine wesentliche Veränderung im Chemismus.

Fall 3. 21. Februar 1891. H. K., 33 Jahre alt, leidet seit 10-15 Jahren an "Ausspeien von Wasser" (darunter versteht Patient das Hochkommen einer grösseren Menge geschmackloser Fllissigkeit aus dem Magen nach dem Munde) jeden Tag oder wenigstens jeden anderen Tag. Zuweilen kam dieses öfter am Tage vor, bis zehn- bis zwölfmal. In der letzten Zeit (etwa seit acht bis neun Monaten) hatte Patient dieses Ausspeien nach jedem Essen. Appetit niemals gut, aber Patient konnte doch jedesmal essen. Als Junge kraftig und nicht mager, seit dem 20. Lebensjahr dagegen mager. Schlaf gut. Stuhlgang einen Tag um den anderen, aber nicht regelmässig. Erbrechen (d. h. Herauskommen der ganzen Mahlzeit) kam ofters wahhrend der letzten 13 Jahre vor. Vor dem Erbrechen hat Patient ein Gefuihl von Druck. Die Untersuchung der Brustorgane ergiebt nichts abnormes; der Magen reicht bis etwa zwei Fingerbreit oberhalb der Symphyse (Gastrodiaphanie und Klatschgerăusch).

22. Februar 1891. Eine Stunde nach dem Probefrühstück: $\mathrm{HCl}=0$, Acid. $=14, \mathrm{Lab}+$.

Diagnose: Dilatatio ventriculi, Gastritis glandularis, chronica, Regurgitatio und beginnende Atrophie.

Nach Elektrisation (direkte Faradisation des Magens, vom 21. Februar bis 26. Mărz Faradisation jeden anderen Tag, wăhrend des April nur einmal wöchentlich) und ab und zu vorgenommener Ausspitlung wurde Patient gleich besser; das Ausspeien horte beinahe ganz auf; es kam nur einmal etwa jeden anderen Tag vor. Der Appetit wurde besser, und Patient fühlte sich kräftiger.

Die hẳufig vorgenommenen Uutersuchungen des Magenchemismus ergaben immer so ziemlich dasselbe Resultat; es fehlte die freie Salzsăure, dagegen waren die Fermente des Magens, Pepsin und Lab vorhanden; die Acidität war stets sehr gering und schwankte von 14-22. Nur einmal (27. Februar 1891) gelang es, eine Stunde nach dem Probefrühstack, nach zehn Minuten langer Faradisation des Magens, wobei Patient beim Verschlucken der Elektrode $100 \mathrm{ccm}$ Wasser getrunken hatte, in dem gewonnenen Mageninhalt freie Salzsäure in Spuren nachzuweisen; die Acidität betrug 22 .

Am Schluss der Behandlung glaubt Patient an Gewicht nicht zugenommen zu haben. Stuhlgang war jetzt jeden Tag vorhanden, und das Ausspeien kam beinahe gar nicht mehr vor. Die erzielte Besserung hielt auch nach Aussetzen der Cur an. Ich sah den Patienten am 3. November 1891, wo er mir diese Mittheilung machte; das Ausspeien ist nicht wieder gekommen, und der Stuhlgang war immer regelmassig.

Ein Fall von Atrophie der Magenschleimhaut bei einem 24 Jahre alten Mann, der bereits durch Ausspülung und geeignete Lebensweise erheblich gebessert war, wurde direkt faradisirt; hier leistete die Faradisation etwa dieselben Dienste wie die Magenausspülung. Die Faradisation in diesem Falle bietet wenig bemerkenswerthes, und ist derselbe wegen seines sonstigen klinischen Interesses bereits an anderem Orte besprochen worden. ${ }^{1}$ )

Während alle eben beschriebenen Fälle (mit Ausnahme des sub 4 angeführten Falles Capitän L. M.) einen Nachlass oder vielmehr ein Verschwinden sämmtlicher subjectiven Krankheitssymptome nach wiederholter Application des faradischen Stromes aufwiesen, konnte bei fünf anderen hauptsächlich an hartnäckigen Gastralgieen leidenden Personen zwar eine leichte Besserung, aber keineswegs ein Verschwinden der Krankheitssymptome notirt werden; in allen diesen Fällen wurde der galvanische Strom, und zwar mit Erfolg angewandt. Die genaue Beschreibung dieser Fălle erfolgt im folgenden Abschnitt.

(Schluss folgt.) 